\title{
Anomalías orofaciales en pacientes con síndrome de Down en una muestra de población chilena
}

\author{
Dra. Lilian Jara S. 1; Dr. Alejandro Ondarza G. ${ }^{\text {; }}$ Dr. José I. Infante L. ${ }^{2}$; \\ Sr. Samuel Gac H. 3; Sr. Jorge González M. 3; Srta. Paulina Salas S. ${ }^{3}$; \\ Sr. Mauricio Santos C. ${ }^{3}$; Sr. Rafael Yáñez A. ${ }^{3}$ \\ Oral anomalies in patients with Down's syndrome \\ and unvelated mental retardation
}

\begin{abstract}
Sixty nine Down's syndrome patients and 68 not associated to Down's syndrome were studied in a chilean school population. In order to determine the oral health a nomalies in Down's syndrome patients, both groups were examined for abnormalities in their gingival tissues, palate configuration and abnotmal development, presence or absence of torus, dentitional types, dental caries, fusion of dental pieces, abnormal dental positions, missing tooth, tongue characteristics and tooth brushing frequency. Our results show important differences with the findings , reported in the literature in Down's syndrome patients of different ethnic orig in; particularly a lower incidence of 'periodontal disease, higher incidence of inflamatory changes in the upper gingivae and different distribution of missing tooth in cases with this abnormality. This differences suggest some ethnic and environmental influences in the somatic expression of Dow'n's syndrome. When compared with other mentally retarded patients, those with Down's syndrome showed higher fuecuencies of abnormal dentat position $(59.42$ vs $32.35 \% \mathrm{p}=0.001$ ) and prognatism (39.21\%vs $5.64, p=0.001)$ respectively.
\end{abstract}

(Key words: Down's syndrome, trisomy 21, dental anomalies, teeth, gingival, periodontal disease, occlusal disharmonies, caries, oral manifestations).

En pacientes con sindrome de Down. (S. Down), se han realizado estudios de prevalencia de caries, enfermedad periodontal, anodoncia, malposición de pjezas dentarias y anomalías de oclusión. Los pacientes presentan mayor susceptibilidad a la paradenciopatias ${ }^{1-7}$ y en algunas series 90 a $96 \%$ de los pacientes presentaba enfermedad periodontal ${ }^{8-11}$, en contraste con sólo $6 \%$ de nif́os controles de edad comparable. La prevalencia de anodoncia ha variado entre $25 \%$ y $93 \% 1,3,10,12-2.4$ y además se han descrito alteraciones de la oclusión $6,11,18$, 24-29. La incidencia de caries dentales en población sajona afectada, por el sindrome es, en cambio baja al compararla con pacientes con otros tipos de retardo mental o sujetos normales $1,5,9,11,25-27,30-37$. Esta baja frecuencia de caries se produce a pesar de la deficiente higiene bucal ${ }^{31}$. En un estudio de incidencia de caries en escolares chilenos con síndrome de Down se encontró, por el contrario, un elevado

1. Departamento de ciencias médico-biológicas y básicas sur. Facultad de Medicina, Universidad de Chìe.

2. Departamento de pediatría y cirugía infantil sur. Facultad de medicina. Universidad de Chile.

3. Alumno carrera Medicina. Universidad de Chile. porcentaje de caries en comparación con to observado en pacientes con retardo mental no asociado al sindrome ${ }^{7}$. La frecuencia de cepillado dental en todos los pacientes examinados fue de tres veces al día. Considerando dichos resultados, se estudiaron las anomalias orales de pacientes con síndrome de Down con el propósito de obtener información que facilite el correcto manejo preventivo y curativo de sus afecciones odontológicas.

\section{MATERIAL Y METODO}

Se examinaron 69 pacientes con síndrome de Down, de la Escuela Especial No 497 y del Centro de Educa. ción Especial para niños Deficitatios. Sesenta y ocho pacientes con retardo mentai no asociado con sínd rome Ae Down, aiumnos de la Escuela Especial 497, constituyeron el grupo control. El procedimiento de examen clinico oral uniforme para todos los individuos, se realizó bajo condiciones adecuadas de iluminación 37 , mediante sondas de caries curvas No 5 y espejos planos No 4 y 5. Todos los exámenes y evaluaciones fueron hechas por un solo examinador. Los datos obtenidos se registraron en una ficha diseñada para este objeto que consideró: estado de la mucosa en ambos maxilares, configuración palatina, presencia o ausencia de torus, tipo de denticion, presencia de caries, piezas dentarias fusionadas, anomalias de posición, ausencia de piezas dentarias, forma y color de los incisivos, alteración del desarrollo mandibular, caracteristicas linguales $y$ fre- 
cuencia de cepillado. St diagnosticó la enfermedad periodontal y el grado de intensidad del daño se evaluó de acjuerdo al índice Periodontal (Pl) de Russell (1956) sugerido para estudios epidemiológicos ${ }^{38}$. Los resultados se expresaron en porcentajes $y$ se realizó anádisis estadístico de los dalos mediante pruebas de chi cuadrado y de Student.

\section{RESULTADOS}

En $51,34 \%$ de los pacientes con sindrome de Down se encontraron alteraciones inflamatorias de la mucosa en el maxilar superior, que en el grupo control se observaron en $41,17 \%$ de la muestra. En el maxilar inferior, dichas alteraciones se encontraron en 36,48\% del grupo Down y en $31,88 \%$ de los controles. Las anomalias dentarias de posición se encontraron en $59,42 \%$ de los pacientes con S. de Down y sólo en $32,35 \%$ de los controles, diferencia estadisticamente significativa $(\mathrm{p}=0,001)$. La mayor incidencia de anomalías de posición se observó en los incisivos centrales y laterales de las arcadas superior e inferior. En las mandibulas las alteraciones en ambos grupos se manifestaron como retrognatia: micrognatia y prognatismo. En $11,76 \%$ de los pacientes con S. de Down y en 7,52\% del grupo control habia micrognatia. El retrognatismo, fue poco frecuente en ambos grupos: 1,96\% en los pacientes con S. de Down, y $1,88 \%$ en el grupo control. El prognatismo, mostró una incidencia notoriamente mayor en los pacientes con $S$. de Down: $39,21 \%$ vs $5,64 \%$ en los controles, diferencia también estadísticamente significativa $(\mathrm{p}=0,001)$. La incidencia de piezas dentarias ausentes también resultó porcentualmente mayor en el grupo con S. de Down en relación al grupo control: $27,45 \%$ vs $18,8 \%$. La ausencia comprometía preferentemente las piezas 10,11 (incisivos superiores), 15,18 y 31 . (piezas posteriores). En cuanto a la oclusión, la mordida estaba alterada en $46,38 \%$ de los pacientes con S. de Down y en $30,88 \%$ en el grupo control, diferencia que no alcanzó significado estadístico. En la Fígura l se tesume la información obtenida, en ella se pueden apreciar las diferencias observadas entre ambos grupos en los parámetros utilizados.

\section{DISCUSION}

Los resultados encontrados en población Down chilena con respecto a algunos aspectos de salud oral, difieren de 10 descrito por otros autores. En relación a enfermedad periodontal su incidencia es marcadamente menor. Este hecho adquiefe máyor significación si se considera que numerosos autores destacan que los pacientes con S. de Down presentan mayor susceptibilidad a las periodonciopatías que la población normal. Otro aspecto intesesante es la mayor frecuencia de gingivitis en el maxilar superior de esta muesträ, en contraposición con lo descrito por los autores citados ${ }^{9}$ quienes encontraron mayor frecuencia en el maxilar inferior. Se ha descrito mayor prevalencia de gingivitis en pacientes institucionalizados con respecto a los que residen en su hogar ${ }^{5}$. El total de los individuos de la muestra Down chilena reside en su hogar y las

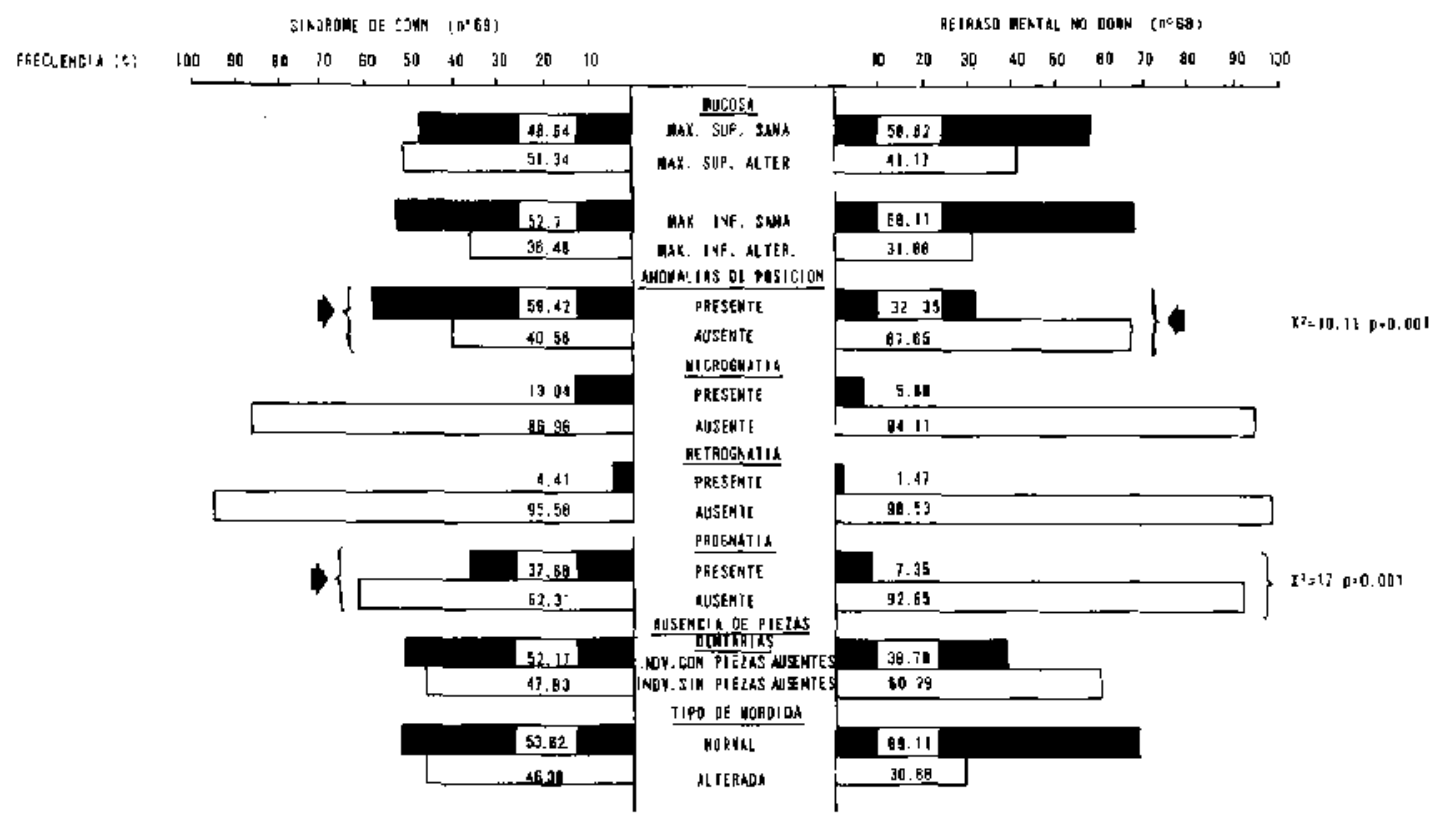

Figura 1. Resumen gráfico de la salud oral en pacientes con síndrome de Down ý niños con retraso mental de otras causas. 
frecuencias son menores. El porcentaje de anodoncia es semejante al descrito por otros autores, existiendo diferencias con respecto al tipo de piezas ausentes ${ }^{19}$. En relación a la oclusión, existen diferencias en los resultados obtenidos y lo descrito en la literatura $11,18,21,24,25,29$. Las discrepancias encontradas al comparar los paráme tros estudiados con los descritos por otros autores, están de acuerdo con la teoría propuesta por Shapiro ${ }^{39}$. Según este autor el estado trisómico aumenta la variabilidad, observándose que para muchos rasgos la población Down es más yariable. Por lo tanto, si bien el S. de Down es el resultado de una alteración numérica a nivel del par 2l, los efectos de la trisomia pueden variar $y$ concurrir factores tales como el origen étnico de las poblaciones y otros de naturaleza ambiental como la calidad de la dieta, hábitos alimentarios u otros. Los resultados encontrados permiten conocer el tipo e incidencia de las anomalías existentes en relación con la salud oral de estos pacientes, lo que debera proyectarse sobre los enfoques de prevención y tratamiento de estos enfermos.

\section{RESUME, N}

Se examinaron 69 pacientes con sindrome de Down de población escolar chilena y 68 pacientes con retardo mental no asociado con sindrome de Down, los que constituyeron el grupo control. Con el objeto de determinar las anomalias existentes en cuanto a salud oral en los enfermos con síndrome de Down, en ambos grupos se analizó estado de la mucosa en ambos maxilares, configuración palatina, presencia o ausencia de torus, tipo de dentición, presencia de caries, piezas dentarias fusionadas, anomalías de posición. ausencia de piezás dentarias, alteración del desarrollo mandibular, características linguales y frecuencia de cepillado. Los resultados obtenidos indican que existen importantes diferencias en los parámetros estudiados, con respecto a 10 indicado en la literatura en pacientes con sínd rome de Down de origen étnico diferente. Las anomalías dentarias de posición y el prognatismo son significativamente más frecuentes en pacientes con sindrome de Down que en niños con retardo mental de otras causas: $59,42 \%$ vs $32,35 \%, p=0,001$ y $39,2\lfloor \%$ vs $5,64 \%, p=0,001$ respectivamente.

\section{REFERENCIAS}

1. Nash, J.A.: citado er Benda C. "The child with mongolism". Grune y Strafton, New York, 1960

2. Cohen, M.M., Sr.: "Pediatric Dentistry". 2nd. Fdition. C.V. Mosby Co. St. Louis, 1961.
3. Cohen, M.M., Sr. and Winer, R.A.: Dental and facial characteristics in Down's syndrome (mongolism). J. Dent. Res. 44: 197, 1965

4. Claycomb, C.K., Summers. G.W., Hall, W.B. and Hart, $R$. W.: Gingival collagen biosynthesis in mongolism. J. Periodont. Res. 5: 30, 1970.

5. Cutress, T.W..: Periodontal disease and oral hygiene in trisomy 21. Arch. Oral. Biol. 16: 1345, 1971.

6. Jensen, G.M., Cleall, J.F. and Yip, S.G.: Dentoalvcolar morphology and devclopmental changes in Down's syndrome (trisomy 21). Am. J. Orthod. 64: $607,1973$.

7. Ondarza, A., Jara, L., Yáñez, R., Saenger, $S . e$ Infonte, J.I.: Incidencia de caries en pacientes con síndrome de Down en una muestra de población chilena. Jornadas Cientificas. Facultad de Medicina, División Ciencias Médicas Sur. R. 8 Agosto, 1985.

8. Cohen, M.M., Sr.: Periodontal distutbances in the mentally subnotmal child. Dent. Clin. North. Am. 4: 483,1960

9. Cohen, M.M., Sr., Winer, R.A. Schwartz, S. and Shtork, $G$ : Oral aspects of mongolism. Part I. Periodontal Disease in Kongolism. Oral Surg. Oral Med. Oral Pathol., 14: 92, 1961.

10. Cohen. M.M. Sr.: Chromosomal Disorders. Dent. Clin. North. Am. 19: 87, 1975.

11. Brown, R.If and Cunningham W.M.: Some dental manifestations of mongolism. Oral Surg. Oral Mod. Oral Pathol. 14: 664, 1961.

12. Dow, R.S.: A prelininary study of periodontoclasia in mongolian children at Polk-State School. Am. J. Ment. Defic. 55: 535, 1951.

13. Ingalls, T.H. and Butler, R.L.: Mongolism - implications of dental anomalies. New Engl. J.'Med. 248: 511,1953

14. Spitzer, $R$. and Robinson, M.L.: Radiological changes in teeth and skull of mental defectives. Br. J. Radiol. 28: 117, 1955.

15. Mc. Millan, R.S. and Kashgoriam. M.: Relation of human abnormalities of structure and function to abnormalities of the dentition. II Mongolism. J Am. Dent. Assoc. 63: 368, 1961 .

16. Silimbani, $C$ : Contributions to the study of dental anomalies in mongolian idiocy. Panminerva Med. 4: 532,1962

17. Barkla, D. . . Congenital absence and fusion in the deciduous dentition in mongols. J. Ment. Defic. Res, 7: 102, 1963.

18. Kessling, E.: "Cranial Morphology in Down's Syndrome". A comparative roentgencephalometric study in adult males. Munksgaard, 1966.

19. Otero, B. y Sznodjer, N.: Agenesia de dientes permanentes en el síndrome de Down (moneolismo). Rev. Asoc. Odont. Argent. 54: 257, 1966.

20. Roche, A.F. and Barkda, D.H.: The development of the dentition in mongols. Aust. Dent. J. 12: 12, 1967.

21. Frostad, W.A. and Cleall, J.F.: A cephalometric and adontometric assesment of the dentofacial morphology in Down's syndrome (Trisomy 21). I.A.D.R. Program and Abstracts of Papers. No. 158. March, 1969.

22. Cohen, M.M. Sr., Blitzer, F.J., Arystas, M.G. and Bonncau, R.M.: Abnormalities of the pernanent dentition in trisomy. J. Dent. Res. 49 (Suppl.) 1386,1970 .

23. Cutress, T.W.: Dental caries in Trisomy 21. Arch, Oral Biol. 16: 1329, 1971.

24. Gosman, S.D. and Vineland, N.J.: Facial developthent in mongolism. Am. J. Orthod. 37: 332, 1951. 
25. Swallow $J N$ : Dental disease in children with Down's syndrome. J. Ment. Defic. Res. 8: 102, 1964.

26. Svatun, B. and Helöe. L.A.: Dental szatus and treatrnent needs among institutionalized mentally subnormal persons in Norway. Comnunity Dent. Oral Epidemiol. 3: 208, 1975

27. Scwlly, $C_{\text {: }}$ Down's syndrome: aspects of dental care. J. Dent. 4: 167, 1976.

28. García, A.f.: Iistudio de la oçusión en niños mongólicos entre 4 y 7 años. Prueba dc Perfeccionamiento clínico. Facultad de Odontología. U. de Chile, 1977.

29. Cohen, M.M. Sr. Arvystas, M.G. and Baum, B.J.: Occlusal disharmonies in trisomy $G$ (Down's syndrone, mongolism). Am. J. Orthod. 58: 367, 1970 .

30. Johnson, N.P., Young, M.A. and Gallios, J.A.: Dental caries expericnce of mongoloid children. ASDC J. Dent. Child. 27: 292, 1960.

31. Witter, R.A. and Cohen, M.M. Sr.: Dental caries in institutionalized nongoloid patients. J. Dent. Res. 40: 661,1961 .
32. Rapaport, J.; Mongolian oligophrenia and dental carics. Rev. Stomatol, Chir. Maxillofac. 64: 207, 1963.

33. Horowitz, H.S., Greek, W.J. and Hoag, O.S.: Study of the provision of dental cate for handicapped children. J. Am. Dent. Assoc. 7 ] : 1398, 1965.

34. Wolf, W.C.: Caries incidence in Down's syndrome (mongolism). J. Wisconsin Den1. Soc. 43: 3, 196 ?

35. Parkin, S.R., Hargreaves, S.A. and Weyman, $f_{\text {.: }}$ Children's dentistry in general practice. II Dental care of physically and mentally handicapped chitdren. Bi. Dent. J. 129: 515, 1970 .

36. Cohen, M.M. Sr. and Cohen, M.M. Sr.: The Oral Manifestations of Trisomy $G$. (Down Syndrome). Birth. Defects. 7: 241, 1971.

37. Orner. G.: Dental caries experience among thildren with Down's syndrome and their sibs. Arch. Oral. Biol, 20: 627, 1975.

38. Russell, A.J.. A system of classification and scoring for prevalence surveys of periodontal divease. I. Dent. Res. 35: 350, 1956.

39. Shapiro, B.L.: Prenatal dental anomaljes in mongolism: comments on the basis and implications of variability. Ann. X.Y. Acad. Sci. 171: 562, 1970 